\title{
New genomic somatic amplifications and deletions in papillary thyroid cancer
}

\author{
Myriem Boufraqech $^{1}$ - Electron Kebebew ${ }^{1}$
}

Received: 14 July 2015/Accepted: 20 July 2015/Published online: 30 July 2015

(C) Springer Science+Business Media New York (outside the USA) 2015

There have been striking changes in the epidemiology of thyroid cancer worldwide with higher incidence rates, with low-risk or clinically less significant papillary thyroid cancer accounting for more than $80 \%$ of new cases each year $[1,2]$. During this change in epidemiology, our knowledge in the genetic and genomic alterations associated with thyroid cancer initiation and or progression has also expanded tremendously [3]. As precision medicine is becoming a reality for some solid malignancies, the application of new knowledge to the management of thyroid cancer is going to be imperative to precisely treat patients based on the genetics/genomics of their tumor, especially when it is found to be associated with disease behavior [4].

The study by Passon and colleagues from Italy reports new findings based on a careful analysis of 27 papillary thyroid cancer samples using high-resolution comparative genomic hybridization [5]. The investigators found that genomic amplifications were higher in high/intermediaterisk group tumors and that genomic deletions were higher in low-risk tumors based on the American Thyroid Association (ATA) risk stratification system. Several of these genomic amplifications and deletions were recurrent (occurred in more than $20 \%$ of tumor samples) and several of the genes in the chromosomal loci with deletions have been implicated to be involved in thyroid cancer or their expression is dysregulated in cancer. The most frequent amplifications that were detected in $2 \mathrm{q} 35,4 \mathrm{q} 26$, and $4 \mathrm{q} 34.1$ contained FN1, PDE5A, and GALNTL6, respectively. The

Electron Kebebew

kebebewe@mail.nih.gov

1 National Cancer Institute, National Institutes of Health, Bethesda, MD, USA 2q35 locus is thought to be a breast cancer susceptibility locus, and FN1 expression was found to be restricted to thyroid cancer samples and absent in benign lesions [6, 7]. Interestingly, $60 \%$ of the cases with $2 \mathrm{q} 35$ amplification were TNM Stage I according to the American Joint Committee on Cancer (AJCC) staging system, whereas more than $70 \%$ were considered intermediate/high risk according to the ATA risk classification system. Although both systems include some of the same variables, the AJCC staging system also includes the patient's age at diagnosis so that patients younger than 45 years even with lymph node and or distant metastases are considered as having only stage I/II disease. Also the ATA risk stratification system includes postoperative serum thyroglobulin level measurement. Thus, these factors likely explain the somewhat discrepant results. The amplification in $4 \mathrm{q} 26$ involving PDE5 was more prevalent in intermediate/highrisk cancers. Interestingly, studies have shown that tumors with high expression level of PDE5 have lower mRNA expression of differentiation markers or thyroid-specific genes (TG, TSHR, TPO, and NIS) [8]. Thus, PDE5 could be involved in thyroid cancer initiation, progression, or even radioiodine resistance. The authors also investigated the $B R A F$ mutation status in the tumors analyzed but found no significant association with genomic amplifications or deletions. Most, but not all, studies have suggested that papillary thyroid cancers with BRAF mutations are more aggressive and that it is one of the most frequent genetic alterations in papillary thyroid cancer $[3,9]$. Passon and colleague did find that 8 of the BRAF V600E mutant samples were in patients with ATA intermediate-risk papillary thyroid cancers. It would be interesting to know if the genomic deletion status in addition to BRAF mutation status could better stratify ATA low-risk from intermediate/high-risk papillary thyroid cancer or even outperform 
the currently used staging or risk classification systems for thyroid cancer with respect to the risk of persistent/recurrent disease. While the study by Passon and associates is an initial investigation, such findings could be used as adjunct prognostic markers that could be translated into making therapeutic decisions if they are validated to be predictors of patient outcome similar to the use of $M Y C N$ amplifications in neuroblastoma [10].

In conclusion, we congratulate Passon and colleagues on their report of new, recurrent chromosomal abnormalities in papillary thyroid cancer which may have important roles in thyroid carcinogenesis and could translate to prognostic markers that can be used to make patient care decisions.

\section{Compliance with ethical standards}

Conflict of Interest The authors have no conflict of interest to report.

\section{References}

1. H.S. Ahn, H.J. Kim, H.G. Welch, Korea's thyroid-cancer "epidemic" - screening and overdiagnosis. New Engl. J. Med. 371(19), 1765-1767 (2014). doi:10.1056/NEJMp1409841

2. D.S. McLeod, A.M. Sawka, D.S. Cooper, Controversies in primary treatment of low-risk papillary thyroid cancer. Lancet 381(9871), 1046-1057 (2013). doi:10.1016/S0140-6736(12)62205-3

3. Cancer Genome Atlas Research, Integrated genomic characterization of papillary thyroid carcinoma. Cell 159(3), 676-690 (2014). doi:10.1016/j.cell.2014.09.050
4. F.S. Collins, H. Varmus, A new initiative on precision medicine. New Engl. J. Med. 372(9), 793-795 (2015). doi:10.1056/ NEJMp1500523

5. N. Passon, E. Bregant, M. Sponziello, M. Dima, F. Rosignolo, C. Durante, M. Celano, D. Russo, S. Filetti, G. Damante, Somatic amplifications and deletions in genome of papillary thyroid carcinomas. Endocrine (2015). doi:10.1007/s12020-015-0592-z

6. O.L. Griffith, A. Melck, S.J. Jones, S.M. Wiseman, Meta-analysis and meta-review of thyroid cancer gene expression profiling studies identifies important diagnostic biomarkers. J. Clin. Oncol. 24(31), 5043-5051 (2006). doi:10.1200/JCO.2006.06.7330

7. Y.L. Fan, Z.J. Guo, P. Zhu, X.J. Yang, X.D. Yang, B. Yu, L.H. $\mathrm{Li}$, Assessing the interactions between the associations of common genetic variants on $2 \mathrm{q} 35$ and $16 \mathrm{q} 12$ with breast cancer risk. Neoplasma 62(2), 315-325 (2015). doi:10.4149/neo_2015_038

8. M. Sponziello, A. Verrienti, F. Rosignolo, R.F. De Rose, V. Pecce, V. Maggisano, C. Durante, S. Bulotta, G. Damante, L. Giacomelli, C.R. Di Gioia, S. Filetti, D. Russo, M. Celano, PDE5 expression in human thyroid tumors and effects of PDE5 inhibitors on growth and migration of cancer cells. Endocrine (2015). doi:10.1007/s12020-015-0586-x

9. M. Xing, A.S. Alzahrani, K.A. Carson, D. Viola, R. Elisei, B. Bendlova, L. Yip, C. Mian, F. Vianello, R.M. Tuttle, E. Robenshtok, J.A. Fagin, E. Puxeddu, L. Fugazzola, A. Czarniecka, B. Jarzab, C.J. O'Neill, M.S. Sywak, A.K. Lam, G. Riesco-Eizaguirre, P. Santisteban, H. Nakayama, R.P. Tufano, S.I. Pai, M.A. Zeiger, W.H. Westra, D.P. Clark, R. Clifton-Bligh, D. Sidransky, P.W. Ladenson, V. Sykorova, Association between BRAF V600E mutation and mortality in patients with papillary thyroid cancer. JAMA 309(14), 1493-1501 (2013). doi:10.1001/jama.2013.3190

10. M. Huang, W.A. Weiss, Neuroblastoma and MYCN. Cold Spring Harbor Perspect. Med. 3(10), a014415 (2013). doi:10.1101/ cshperspect.a014415 